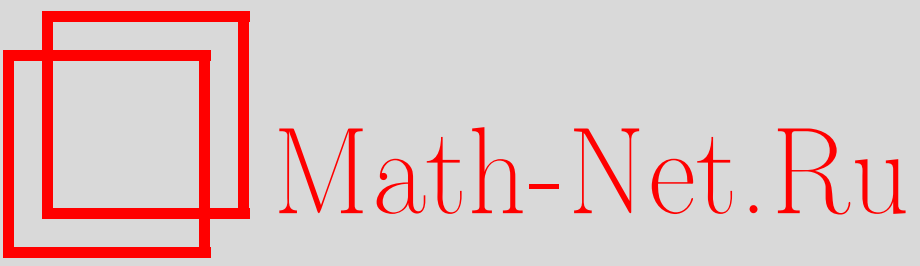

Ю. В. Даревская, О некоторых алгебраических свойствах обобщенных многочленов Чебышёва, УМH, 2003, том 58, выпуск 1, 181-182

DOI: https://doi.org/10.4213/rm598

Использование Общероссийского математического портала Math-Net.Ru подразумевает, что вы прочитали и согласны с пользовательским соглашением

http://www.mathnet.ru/rus/agreement

Параметры загрузки:

IP: 54.162 .27 .143

26 апреля 2023 г., 15:25:19 


\title{
О НЕКОТОРЫХ АЛГЕБРАИЧЕСКИХ СВОЙСТВАХ ОБОБЩЕННЫХ МНОГОЧЛЕНОВ ЧЕБЫШЁВА
}

\author{
Ю. В. ДАРЕВСКАЯ
}

В теории так назьваемых детских рисунков рассматриваются обобщенные многочлены Чебышёва (ОМЧ) [1]. По определению комплексньй многочлен $P$ называется обобщенным многочленом Чебышёва, если для некоторых $c_{+}$и $c_{-}$прообраз отрезка $\left[c_{-}, c_{+}\right]$является деревом [2]. Известно, что приведенное выше определение равносильно ряду чисто алгебраических условий, эквивалентных между собой [3]. Это дает возможность определить обобщенные многочлены Чебышёва над произвольными полями и кольцами. В настоящей заметке за определение обобщенного многочленв Чебышёва над алгебраически замкнутым полем примем следующее (о других эквивалентных определениях см., например, [3]).

ОПредЕлениЕ. Пусть $K$ - алгебраически замкнутое поле. Многочлен $P \in K[z]$, char $K \nmid$ $\operatorname{deg} P$, называется обобщенным многочленом Чебышёва над полем $K$, если существуют $c_{+}, c_{-} \in K$ такие, что все критические значения многочлена $P$ содержатся в множестве $\left\{c_{+}, c_{-}\right\}$. Под критическими значениями подразумевается множество critval $P=\{P(c) \mid$ $\left.P^{\prime}(c)=0\right\}$. Класс таких многочленов обозначается ОМЧ $(K)$.

В качестве естественного обобщения в случае целостного кольца примем следующее

ОПРЕДЕЛЕНИЕ. Пусть $R$ - целостное кольцо, $k$ его поле частных, $\bar{k}$ - алгебраическое замыкание $k$. Тогда

$$
\mathrm{OM \Psi}(R):=\{P \mid P \in R[z] \cap \mathrm{OM \Psi}(\bar{k}), \operatorname{critval}(P) \in R\} .
$$

Некоторые серии обобщенных многочленов Чебышёва обладают следующим свойством лакунарности: степени входящих в них одночленов образуют арифметическую прогрессию с разностью, большей единицы. Например, этим свойством обладают обычные многочлены Чебышёва. Оказывается, многочлен над полем $K$ нулевой характеристики может лежать в ОМЧ $(K)$ лишь при некоторых ограничениях на разность этой прогрессии, о чем и говорится в первой части этой заметки.

Упомянутое понятие лакунарности многочлена можно описать более детально с помощью следующей леммы.

Лемма. Пусть $P(z) \in R[z]-$ многочлен, $R$ - кольцо, $P$ представлен в виде

$$
P(z)=z^{r} S\left(z^{k}\right),
$$

где $S(z)$ - многочлен, $r \in \mathbb{N} \cup\{0\}, k \in \mathbb{N}, k-$ максимально возмо жное, $0 \leqslant r<k$. Тогда $r$ есть остаток от деления $\operatorname{deg} P$ на $k$.

В соответствии с представлением $(*)$ возможны следующие ситуации:

1а) $r=0, k=1$ - многочлен не является лакунарным;

1b) $r=0, k \neq 1$ - многочлен является лакунарным (и, более того, является композиционно приводимым);

2) $r \neq 0$ - многочлен является лакунарным (возможно, при этом не являясь композиционно приводимым).

Ясно, что в случае $P \in K[z]$, где $K$ - поле, любая из ситуаций 1a), 1b), 2) может реализовываться. Оказьвается, что при дополнительном условии $P \in \mathrm{OMЧ}(K)$ это не так, а именно имеет место следующая теорема.

Теорема 1. Пусть $P \in \mathrm{OMЧ}(K), K$ - поле, char $K=0, P$ представлен в виде $(*)$. Тогда либо $r=0$, либо $k$ - четное $u r=k / 2$.

Теорема не имеет места в поле $F, \operatorname{char} F>0$, что вытекает из следующего утверждения. 
УТВеРЖДЕНИЕ. Пусть $P \in \mathbf{F}_{p}[z]$ имеет следующее представление $(*): P=z^{r} S\left(z^{s p}\right)$, $r>0,(r, p)=1$. Тогда для любого $s \in \mathbb{N}, P \in \mathrm{OMЧ}\left(\mathbf{F}_{p}\right)$.

Доказательство теоремы 1 опирается на следующее утверждение.

УтвеРЖДЕнИЕ 1. Пусть $K-$ поле, char $K=0, P-$ обобщенный многочлен Чебьшёва, $P \in K[z]$. Тогда $P \in \mathrm{OMЧ}(L)$, где $L-\operatorname{noле~}(L: K) \leqslant 2$.

Оказьвается, с представлением $(*)$ также связано свойство коэффициентов многочлена $P \in \mathrm{OMЧ}(\mathbf{Z})$, представляющее интерес в связи с вопросом о принадлежности классу ОМЧ $(\mathbf{Z})$ многочлена, полученного из $P$ афффинной заменой. В частности, следствие теоремы 2 показьвает, что в случае $r=0$ с помощью афффинной замены можно добиться того, чтобы коэффициенты многочлена, лежащего в ОМЧ(Z), не содержали случайных общих множителей.

TeOpema 2. Пусть $P \in \mathrm{OMЧ}(\mathbb{Z}), \operatorname{deg} P=n$, critval $P=\left\{c_{-} ; c_{+}\right\}$. Пусть также $в$ представлении $P$ в виде $(*), S(z)=z^{l}+a_{1} z^{l-1}+\cdots+a_{l-1} z+a_{l}, a r=0, p-$ nростое, $(\operatorname{deg} P, p)=1$. Тогда если для любого $i$ выполнено $p^{i} \mid a_{i}$, то $p^{n / k} \mid c_{ \pm}$. В частности, в случае $k=1$ если для любого $i$ выполняется $p^{i} \mid a_{i}$, mо $p^{n} \mid c_{ \pm}$.

СледСтвиЕ. Пусть выполнень условия предьдущей теоремы. Пусть также $Q=$ $1 / p^{n / k} P(p z)$. Тогда $Q \in \mathrm{OMЧ}(\mathbf{Z})$.

Теорема 2 доказывается с помощью следующего утверждения.

УТВЕРЖДЕНИЕ 2. Пусть $K$ - алгебраически замкнутое поле, $P \in \mathrm{OMЧ}(K), c_{+}, c_{-}-$ критические значения $P$. Тогда множество корней многочлена $\operatorname{Discr}(P-c)$ как многочлена от переменной с содержится в множестве $\left\{c_{+}, c_{-}\right\}$.

Автор выражает благодарность Г.Б. Шабату за постоянное внимание и множество полезных советов.

\section{СПИСОК ЛИТЕРАТУРЫ}

[1] G. Shabat, A. Zvonkin // Contemp. Math. 1994. V. 178. P. 233-275. [2] A. Grothendieck // London Math. Soc. Lecture Note Ser. 1997. V. 242. P. 5-48; 243-283. [3] A. M. Baшeвник // Функц. анализ и его прил. 2001. Т. 35. № 3. С. 77-79.

Московский государственный

Принято редколлегией университет им. М. В. Ломоносова 